\title{
ESTRUTURA DE UM CERRADO STRICO SENSU NA GLEBA CERRADO PÉ-DE-GIGANTE, SANTA RITA DO PASSA QUATRO, SP
}

\author{
Alessandra Tomaselli Fidelis ${ }^{1,3}$ \\ Silvana Aparecida Pires de Godoy ${ }^{2}$
}

Recebido em 24/10/2002. Aceito em 08/05/2003

\begin{abstract}
RESUMO - (Estrutura de um Cerrado stricto sensu na Gleba Cerrado Pé-de-Gigante, Santa Rita do Passa Quatro, SP). O Cerrado ocupa aproximadamente $23 \%$ do território brasileiro e $70 \%$ do bioma correspondem a cerrado stricto sensu (s.s.), sendo relevantes os estudos que buscam o entendimento da estrutura da vegetação nessas áreas. Com esse objetivo, foram estudadas cinco parcelas $(10 \times 25 \mathrm{~m})$ em um hectare de cerrado s.S., amostrando-se todos os indivíduos com perímetro no nível do solo igual ou acima de $3 \mathrm{~cm}$ (exceto lianas e indivíduos mortos). Alguns parâmetros fitossociológicos foram analisados, assim como a distribuição de classes de diâmetro dos indivíduos amostrados e a estrutura vertical. Foram amostradas 1.747 indivíduos, distribuídos em 75 espécies, pertencentes a 31 famílias. A densidade total absoluta encontrada foi de 13.976 ind.ha ${ }^{-1}$ e a área basal total, de 4,902 $\mathrm{m}^{2}$. Leguminosae foi a família com o maior número de espécies (16). As espécies que apresentaram os maiores valores de Índice do Valor de Importância (IVI) foram Anadenanthera falcata (Benth.) Altschul, Myrcia guianensis (Aubl.) DC., Xylopia aromatica (Lm.) Mart., Ouratea spectabilis (Mart.) Engl. e Pouteria ramiflora (Mart.) Radlk. O Índice de Shannon encontrado foi de 3,623. A distribuição de classes de diâmetro apresentou curva na forma de "J" invertido, estando a maioria dos indivíduos na primeira classe. A área estudada não apresentou estratos bem definidos, estando a maioria dos indivíduos entre 1 e $3 \mathrm{~m}$ de altura.
\end{abstract}

Palavras-chaves - cerrado, fitossociologia, fisionomia, estrutura vertical, classes de diâmetro

ABSTRACT - (Structure of cerrado stricto sensu at the Glebe Cerrado Pé-de-Gigante, Santa Rita do Passa Quatro, SP). The cerrado covers about $23 \%$ of the Brazilian territory, and $70 \%$ of the cerrado area is stricto sensu physiognomy. To promote a better understanding of its physiognomic structure the cerrado s.s. study is of vital importance. Therefore, five plots $(10 \times 25 \mathrm{~m})$ were established in one hectare area of cerrado s.s. and all vascular plants with stem perimeter at ground level $\geq 3 \mathrm{~cm}$ (except vines and dead individuals) were sampled. The distribution of diameter classes and vertical structure, as well as the phytosociological parameters were analyzed. We recorded 1,747 individuals, distributed in 75 species belonging to 31 different families were sampled. The absolute density and the basal area were 13,976 ind.ha ${ }^{-1}$ and 4,902 $\mathrm{m}^{2}$, respectively. Leguminosae showed the highest number of species (16). The species Anadenanthera falcata (Benth.) Altschul, Myrcia guianensis (Aubl.) DC., Xylopia aromatica (Lm.) Mart., Ouratea spectabilis (Mart.) Engl. and Pouteria ramiflora (Mart.) Radlk. showed the

1 Bolsista de Iniciação Científica, CNPq

2 Setor de Botânica, Departamento de Biologia, Faculdade de Filosofia, Ciências e Letras de Ribeirão Preto/USP, Av. Bandeirantes 3900, CEP 14040-901, Ribeirão Preto, SP, Brasil

3 Autor para correspondência: atfidelis@gmx.net 
highest VI. The Shannon's Index was 3,623. The distribution of diameter classes showed a curve in reverted-“J", most of the individuals being in the first class. There were no well defined vegetation strata, showing most of the individuals between 1 and $3 \mathrm{~m}$ in height.

Key words - cerrado, phytosociology, physiognomy, vertical structure, diameter classes

\section{Introdução}

O Cerrado ocupa $23 \%$ do território brasileiro, estendendo-se da margem da Floresta Amazônica até os Estados de São Paulo e Paraná (Ratter \& Dargie 1992; Oliveira-Filho \& Ratter 1995; Ratter et al. 1997), tratando-se do segundo maior bioma do país, superado apenas pela Floresta Amazônica (Ribeiro \& Walter 1998). Esta enorme área é ocupada por um complexo vegetacional que inclui diferentes fisionomias, determinadas primeiramente pela ação do fogo ou pela distribuição dos tipos de solo (Coutinho 1982), ou pela combinação da ação dos fatores clima, solo, disponibilidade de água e nutrientes, geomorfologia e topografia, latitudes, pastejo e impacto de atividades antrópicas (Ribeiro \& Walter 1998). Coutinho (1978) considerou as fisionomias campo sujo, campo cerrado e cerrado stricto sensu como sendo os verdadeiros ecótonos, enquanto as formas extremas estariam representadas pelo campo limpo e cerradão.

A flora do Cerrado é muito antiga (Cretáceo) e os autores divergem quanto ao número de espécies que a compõe. Para Ratter \& Dargie (1992) seria algo em torno de 700 espécies de árvores e arbustos de grande porte. Mendonça et al. (1998) relatam 6.671 táxons nativos, sendo 267 pteridófitas, duas gimnospermas e 6.060 angiospermas. A última estimativa (Castro et al. 1999) mostra o máximo de 2.000 espécies arbóreas e 5.250 espécies herbáceas e subarbustivas, portanto flora muito mais rica do que se pensava inicialmente.

Entretanto, nos últimos 25 anos, o Cerrado vem recebendo ação direta do desenvolvimento da agricultura (Ratter et al. 1996; 1997). Pivello \& Coutinho (1996) afirmam que atualmente, quase todo o ambiente de cerrado está sob intensa pressão humana e não é mais natural.
Assim sendo, deve-se envidar esforços no sentido de fornecer informações que possam contribuir para o conhecimento e subsidiar ações de preservação dos fragmentos existentes.

A condução de estudos florísticos e fitossociológicos nestas áreas é de fundamental importância para o conhecimento da distribuição das espécies e de seu relacionamento com o ambiente (Silva Júnior et al. 1987), além de poderem acrescentar informações sobre possíveis afinidades entre as espécies ou grupos de espécies, podendo tais associações facilitar a compreensão da distribuição das fitofisionomias do cerrado (Ribeiro et al. 1985).

Os Cerrados remanescentes no Estado de São Paulo estão em áreas disjuntas que sobreviveram à agricultura e ao pastoreio (Durigan et al. 1994); e, devido ao seu enorme valor e ao acelerado processo de destruição, urge que medidas sejam tomadas para a preservação de tais remanescentes. Contudo, não são muito numerosos os estudos fitossociológicos nos Cerrados de São Paulo, destacando-se alguns como: Piccolo et al. (1971); SilberbauerGottsberger \& Eiten (1983); Durigan et al. (1987); Ratter et al. (1988); Pagano et al. (1989); Toledo Filho et al. (1989).

Considerando o exposto, o presente estudo visa analisar a estrutura de uma área de cerrado stricto sensu, localizada na Gleba Cerrado Pé-de-Gigante, com os objetivos de fornecer dados que possam contribuir para o conhecimento e a preservação destes remanescentes no Estado de São Paulo.

\section{Material e métodos}

O estudo foi realizado em um hectare de Cerrado stricto sensu, localizado na Gleba Cerrado Pé-de-Gigante, município de Santa Rita 
do Passa Quatro, Estado de São Paulo (21 ${ }^{\circ} 36-38^{\prime} \mathrm{S}$ e $\left.47^{\circ} 36-39^{\prime} \mathrm{W}\right)$. A Gleba tem $1.225 \mathrm{ha}$, em altitudes que variam de 590 a $740 \mathrm{~m}$, em solos do tipo Latossolo Vermelho Escuro associados ao Latossolo Vemelho Amarelo e às Areias Quartzosas, e apresenta as seguintes fitofisionomias de Cerrado: campo sujo, campo cerrado, cerrado stricto sensu (dominante) e cerradão, estando circundada por eucaliptos e plantações de cítricos e cana-de-açúcar (Bitencourt et al. 1997; Pivello et al. 1998; 1999). O clima da região enquadra-se na categoria Cwag (Köppen 1948), ou seja, temperado, macrotérmico, moderadamente chuvoso e com inverno seco não rigoroso.

$\mathrm{Na}$ área escolhida de 1 ha foram lançadas, aleatoriamente, cinco parcelas de $25 \times 10 \mathrm{~m}$. Optou-se pelo método de parcelas, pois além de eficiente na caracterização de formações vegetais naturais (Curtis \& McIntosh 1950), possibilita inventariar diferentes estratos vegetais. O levantamento de dados abrangeu o período de maio/1998 a junho/1999, amostrando-se todos os indivíduos com perímetro a altura do solo (PAS) igual ou maior que $3 \mathrm{~cm}$, excluídas as lianas e os indivíduos mortos.

Os parâmetros fitossociológicos foram calculados segundo Martins (1993), e os índices de diversidade de Shannon e de Eqüabilidade, segundo Magurran (1988). Além disso, para obter melhor entendimento da estrutura da vegetação na área, foi elaborado um histograma de freqüência de classes de diâmetro (classes fixas de $4,5 \mathrm{~cm}$ ) para todos os indivíduos da área. A estratificação foi avaliada através da construção de um histograma e de um gráfico do espaço vertical ocupado pelas espécies amostradas.

\section{Resultados e discussão}

Foram amostrados 1.747 indivíduos, pertencentes a 75 espécies, distribuídas em 55 gêneros e 31 famílias (uma indeterminada) (Tab. 1). Leguminosae apresentou o total de 16 espécies (Caesalpinioideae - seis, Faboideae - cinco e Mimosoideae - cinco), sendo a família com o maior número de espécies. Myrtaceae apresentou oito espécies, seguida de Annonaceae, Malpighiaceae, Melastomataceae e Vochysiaceae (quatro espécies cada). Essas seis famílias contribuíram com $53,34 \%$ do total de espécies amostradas, merecendo destaque na estrutura desta vegetação. Ressalta-se que 18 famílias foram representadas por apenas uma espécie.

Trabalhos sobre cerrados brasileiros também apontaram a importância da família Leguminosae na composição e estrutura da comunidade vegetal (Heringer et al. 1977; Toledo Filho et al. 1984; 1989; Oliveira-Filho et al. 1989; Leitão Filho 1992; Felfili et al. 1993, Felfili \& Silva Júnior 1993; Guarim-Neto et al. 1994; Rizzini 1997; Castro et al. 1998; Mendonça et al. 1998; Uhlman et al. 1998). A importância desta família também já foi ressaltada em trabalhos sobre matas como por exemplo Carvalho et al. (1995); Dias et al. (1998), Terborgh \& Andresen (1998), Tabarelli \& Mantovani (1999) e van den Berg \& OliveiraFilho (2000).

Martins (1993) afirmou que o predomínio de Leguminosae pode estar relacionado à presença de nódulos radiculares, que funcionam como mecanismo de retenção e transferência de nutrientes (nitrogênio) no ecossistema.

Desta forma, pode-se afirmar que a família Leguminosae é de extrema importância, não só na estrutura da vegetação dos cerrados, como também na de florestas e matas, sendo a preservação desta fundamental para a dinâmica populacional destas comunidades.

Ratter et al. (1997) afirmaram que em muitas áreas de cerrado, Vochysiaceae seria a família dominante, principalmente devido à abundância de três espécies de Qualea. O gênero Qualea apresentou na presente análise o maior número de espécies (quatro): $Q$. dichotoma, $Q$. grandiflora, $Q$. multiflora e $Q$. parviflora e, de acordo com Heringer et al. (1977), $Q$. grandiflora e $Q$. parviflora são espécies 
Tabela 1 . Parâmetros fitossociológicos das espécies com PAS igual ou maior que $3 \mathrm{~cm}$, em cerrado stricto sensu na ARIE Cerrado Pé-de-Gigante, Santa Rita do Passa Quatro, SP, em ordem decrescente de IVI. NI - número de indivíduos por espécie; AB - área basal $\left(\mathrm{m}^{2}\right)$; FR - Freqüência Relativa; DR - Densidade Relativa (\%); DoR - Dominância Relativa (\%); IVI - Índice do Valor de Importância.

\begin{tabular}{|c|c|c|c|c|c|c|}
\hline Espécies & NI & $\mathrm{AB}$ & FR & DR & DoR & IVI \\
\hline $\begin{array}{l}\text { 1. Anadenanthera peregrina (L.) Speg. var. falcata } \\
\text { (Benth.) Altschul }\end{array}$ & 112 & 1,5553 & 2,15 & 6,41 & 31,73 & 40,28 \\
\hline 2. Myrcia guianensis (Aubl.) DC. & 149 & 0,3572 & 2,15 & 8,53 & 7,29 & 17,96 \\
\hline 3. Xylopia aromatica (Lm.) Mart. & 96 & 0,2163 & 2,15 & 5,50 & 4,41 & 12,05 \\
\hline 4. Ouratea spectabilis (Mart.) Engl. & 59 & 0,2581 & 2,15 & 3,38 & 5,27 & 10,79 \\
\hline 5. Pouteria ramiflora (Mart.)Radlk. & 82 & 0,1768 & 1,72 & 4,69 & 3,61 & 10,02 \\
\hline 6. Duguetia furfuracea (A. St-Hil.) Benth. \& Hook. & 121 & 0,0342 & 2,15 & 6,93 & 0,70 & 9,77 \\
\hline 7. Copaifera langsdorffii Desf. & 39 & 0,2191 & 2,15 & 2,23 & 4,47 & 8,85 \\
\hline 8. Byrsonima intermedia A. Juss. & 97 & 0,0303 & 2,15 & 5,55 & 0,62 & 8,32 \\
\hline 9. Tabebuia ochracea (Cham.) Standl. & 40 & 0,1468 & 2,15 & 2,29 & 2,99 & 7,43 \\
\hline 10. Myrcia bella Cambess. & 56 & 0,0958 & 2,15 & 3,21 & 1,95 & 7,31 \\
\hline 11. Myrcia uberavensis O. Berg. & 46 & 0,1127 & 2,15 & 2,63 & 2,30 & 7,08 \\
\hline 12. Caryocar brasiliense Cambess. & 20 & 0,1968 & 1,72 & 1,14 & 4,01 & 6,88 \\
\hline 13. Qualea dichotoma (Mart.) Warm. & 22 & 0,1678 & 2,15 & 1,26 & 3,42 & 6,83 \\
\hline 14. Couepia grandiflora (Mart. \& Zucc.) Benth. & 41 & 0,1102 & 1,72 & 2,35 & 2,25 & 6,31 \\
\hline 15. Bauhinia rufa (Bong.) Steud. & 56 & 0,0291 & 2,15 & 3,21 & 0,59 & 5,95 \\
\hline 16. Aspidosperma tomentosum Mart. & 37 & 0,0725 & 2,15 & 2,12 & 1,48 & 5,74 \\
\hline 17 - Annona dioica A. St-Hil. & 56 & 0,0179 & 2,15 & 3,21 & 0,37 & 5,72 \\
\hline 18. Toulicia tomentosa Radlk. & 57 & 0,0249 & 1,72 & 3,26 & 0,51 & 5,49 \\
\hline 19. Campomanesia pubescens (DC.) O. Berg & 47 & 0,0123 & 2,15 & 2,69 & 0,25 & 5,09 \\
\hline 20. Erythroxylum suberosum A. St-Hil. & 19 & 0,1162 & 1,29 & 1,09 & 2,37 & 4,75 \\
\hline 21. Qualea multiflora Mart. & 26 & 0,0497 & 2,15 & 1,49 & 1,01 & 4,65 \\
\hline 22. Ficus citrifolia Mill. & 27 & 0,0407 & 2,15 & 1,55 & 0,83 & 4,52 \\
\hline 23. Dalbergia miscolobium Benth. & 3 & 0,1821 & 0,43 & 0,17 & 3,71 & 4,32 \\
\hline 24. Eugenia punicifolia (Kunth) DC. & 19 & 0,0439 & 2,15 & 1,09 & 0,90 & 4,13 \\
\hline 25. Didymopanax vinosum (Cham. \& Schltdl.) Marchal & 29 & 0,0138 & 2,15 & 1,66 & 0,28 & 4,09 \\
\hline 26. Rourea induta Planch & 37 & 0,0086 & 1,72 & 2,12 & 0,18 & 4,01 \\
\hline 27. Senna rugosa (G. Don) Irwin \& Barneby & 29 & 0,0097 & 2,15 & 1,66 & 0,20 & 4,00 \\
\hline 28. Ocotea pulchella Mart. & 19 & 0,0974 & 0,86 & 1,09 & 1,99 & 3,93 \\
\hline 29. Gochnatia pulchra Cabrera & 26 & 0,0081 & 2,15 & 1,49 & 0,17 & 3,80 \\
\hline 30. Qualea grandiflora Mart. & 19 & 0,0269 & 2,15 & 1,09 & 0,55 & 3,78 \\
\hline 31. Pouteria torta (Mart.) Radlk. & 15 & 0,0224 & 2,15 & 0,86 & 0,46 & 3,46 \\
\hline 32. Miconia albicans Triana & 21 & 0,0053 & 2,15 & 1,20 & 0,11 & 3,46 \\
\hline 33. Tocoyena formosa (Cham. \& Schltdl.) K. Schum. & 18 & 0,0302 & 1,72 & 1,03 & 0,62 & 3,36 \\
\hline 34. Vatairea macrocarpa (Benth.) Ducke & 21 & 0,0208 & 1,72 & 1,20 & 0,42 & 3,34 \\
\hline 35. Diptychandra aurantiaca Tul. & 7 & 0,0926 & 0,86 & 0,40 & 1,89 & 3,15 \\
\hline 36. Stryphnodendron obovatum Benth. & 12 & 0,0345 & 1,72 & 0,69 & 0,70 & 3,11 \\
\hline 37. Annona coriacea Mart. & 15 & 0,0137 & 1,72 & 0,86 & 0,28 & 2,85 \\
\hline 38. Leandra lacunosa Cogn. & 10 & 0,0021 & 2,15 & 0,57 & 0,04 & 2,76 \\
\hline 39. Austroplenckia populnea (Reissek) Lund. & 14 & 0,0242 & 0,86 & 0,80 & 0,49 & 2,15 \\
\hline 40. Machaerium acutifolium Vogel & 5 & 0,0481 & 0,86 & 0,29 & 0,98 & 2,13 \\
\hline 41. Guapira noxia (Netto) Lundell & 4 & 0,0291 & 1,29 & 0,23 & 0,59 & 2,11 \\
\hline 42. Bredemeyera floribunda Willd. & 10 & 0,0285 & 0,86 & 0,57 & 0,58 & 2,01 \\
\hline 43. Eupatorium squalidum A. DC. & 9 & 0,0022 & 1,29 & 0,52 & 0,04 & 1,85 \\
\hline 44. Tapirira guianensis Aubl. & 9 & 0,0011 & 1,29 & 0,52 & 0,02 & 1,83 \\
\hline 45. Eugenia bimarginata DC. & 7 & 0,0051 & 1,29 & 0,40 & 0,10 & 1,79 \\
\hline 46. Byrsonima coccolobifolia A. Juss. & 5 & 0,0051 & 1,29 & 0,29 & 0,10 & 1,68 \\
\hline
\end{tabular}


Tabela 1 (continuação)

\begin{tabular}{|c|c|c|c|c|c|c|}
\hline Espécies & NI & $\mathrm{AB}$ & FR & DR & DoR & IVI \\
\hline 47. Sapium glandulatum (Vell.) Pax & 5 & 0,0046 & 1,29 & 0,29 & 0,09 & 1,67 \\
\hline 48. Hyptis reticulata Mart. & 6 & 0,0014 & 1,29 & 0,34 & 0,03 & 1,66 \\
\hline 49. Bowdichia virgilioides Kunth in HBK. & 4 & 0,0225 & 0,86 & 0,23 & 0,46 & 1,55 \\
\hline 50. Chamaechrista debilis (Vogel) Irwin \& Barneby & 3 & 0,0005 & 1,29 & 0,17 & 0,01 & 1,47 \\
\hline 51. Stryphnodendron adstringens (Mart.) Coville & 5 & 0,0139 & 0,86 & 0,29 & 0,28 & 1,43 \\
\hline 52. Miconia stenostachya A. DC. & 7 & 0,0033 & 0,86 & 0,40 & 0,07 & 1,33 \\
\hline 53. Eriotheca gracilipes (K. Schum.) A. Robyns & 5 & 0,0077 & 0,86 & 0,29 & 0,16 & 1,30 \\
\hline 54. Qualea parviflora Mart. & 4 & 0,0082 & 0,86 & 0,23 & 0,17 & 1,25 \\
\hline 55. Heteropterys byrsonimifolia A. Juss. & 4 & 0,0011 & 0,86 & 0,23 & 0,02 & 1,11 \\
\hline 56. Lippia salviifolia Cham. & 4 & 0,0008 & 0,86 & 0,23 & 0,02 & 1,10 \\
\hline 57. Rudgea viburnoides Benth. & 3 & 0,0007 & 0,86 & 0,17 & 0,01 & 1,04 \\
\hline 58. Pterodon pubescens Vogel & 5 & 0,0056 & 0,43 & 0,29 & 0,11 & 0,83 \\
\hline 59. Styrax ferrugineus Nees \& Mart. & 1 & 0,0147 & 0,43 & 0,06 & 0,30 & 0,79 \\
\hline 60. Plathymenia reticulata Benth. & 1 & 0,0067 & 0,43 & 0,06 & 0,14 & 0,62 \\
\hline 61. Miconia ligustroides Naudin & 3 & 0,0008 & 0,43 & 0,17 & 0,02 & 0,62 \\
\hline 62. Eugenia livida $\mathrm{O}$. Berg. & 2 & 0,0034 & 0,43 & 0,11 & 0,07 & 0,61 \\
\hline 63. Pouteria subcaerulea Pierre ex Dubard & 3 & 0,0003 & 0,43 & 0,17 & 0,01 & 0,61 \\
\hline 64. Diospyros hispida A. DC. & 2 & 0,0015 & 0,43 & 0,11 & 0,03 & 0,57 \\
\hline 65. Palicourea rigida Benth. & 2 & 0,0009 & 0,43 & 0,11 & 0,02 & 0,56 \\
\hline 66. Hancornia speciosa Gomes & 1 & 0,0029 & 0,43 & 0,06 & 0,06 & 0,55 \\
\hline 67. Stryphnodendron polyphyllum Benth. & 1 & 0,0018 & 0,43 & 0,06 & 0,04 & 0,52 \\
\hline 68. Hymenaea stigonocarpa Mart. ex Hayne & 1 & 0,0008 & 0,43 & 0,06 & 0,02 & 0,50 \\
\hline 69. Gochnatia barrosoi Cabrera & 1 & 0,0005 & 0,43 & 0,06 & 0,01 & 0,50 \\
\hline 70. Aegiphila lhotzkiana Cham. & 1 & 0,0004 & 0,43 & 0,06 & 0,01 & 0,49 \\
\hline 71. Connarus suberosus Planch. & 1 & 0,0003 & 0,43 & 0,06 & 0,01 & 0,49 \\
\hline 72. Anacardium humile A. St-Hil & 1 & 0,0002 & 0,43 & 0,06 & 0,00 & 0,49 \\
\hline 73. Byrsonima crassa Nied. & 1 & 0,0001 & 0,43 & 0,06 & 0,00 & 0,49 \\
\hline 74. Indeterminada 1 & 1 & 0,0001 & 0,43 & 0,06 & 0,00 & 0,49 \\
\hline 75. Psidium cinereum Mart. ex DC. & 1 & 0,0001 & 0,43 & 0,06 & 0,00 & 0,49 \\
\hline Total & 1.747 & 4,902 & 100,00 & 100,00 & 100,00 & 300,00 \\
\hline
\end{tabular}

comuns no Planalto Central. A riqueza e a importância deste gênero foram observadas em trabalhos sobre áreas de cerrado no Mato Grosso (Guarim-Neto et al. 1994), Piauí (Castro et al. 1998) e em São Paulo (Leitão Filho, 1992; Toledo Filho et al. 1984), evidenciando que este gênero faz parte da flora de várias áreas de cerrado em todo o Brasil. Além de Qualea, outros gêneros considerados comuns nos cerrados brasileiros foram amostrados no presente trabalho, tais como: Bowdichia, Tabebuia, Byrsonima, Miconia, Eugenia, Annona, Aspidosperma, Myrcia, Didymopanax, Ocotea, Anacardium, Bauhinia, Caryocar, Erythroxylum, Copaifera, Xylopia, Machaerium, Pouteria e Tocoyena
(Heringer et al. 1977; Rizzini 1997; Ratter et al. 1996).

A Área Basal Total (ABT) foi de 4,902 $\mathrm{m}^{2}$, e a Densidade Total Absoluta (DTA) foi de 13.976 ind.ha ${ }^{-1}$ (Tab. 1), sendo esta maior do que aquelas verificadas em outros estudos realizados em cerrados stricto sensu (Toledo Filho et al. 1989; Felfili \& Silva Júnior 1993; Durigan et al. 1994; Felfili et al. 1998). Este valor mais alto de DTA comparado a valores obtidos em outros trabalhos está certamente relacionado ao critério de inclusão, uma vez que muitas herbáceas e subarbustos foram amostrados neste estudo.

$\mathrm{O}$ valor do Índice de diversidade de Shannon (H') encontrado foi de 3,623 e o de 
Eqüabilidade (E), 0,839. O valor de H' diverge discretamente dos encontrados por Durigan et al. (1994) e Toledo Filho (1989), 3,08 e 3,51, respectivamente.

Das 75 espécies (Tab. 1) amostradas, 25 perfizeram 53,75\% da Freqüência Relativa (FR) total. Entre elas, encontram-se aquelas mais abundantes como Myrcia guianensis (149) e Duguetia furfuracea (121), e outras com baixo número de indivíduos, como Leandra lacunosa (10) e Pouteria torta (15). Estas espécies, apesar do baixo número de indivíduos, foram amostradas em todas as parcelas, podendo estar, deste modo, mais distribuídas na área, afirmação passível de comprovação através de um estudo de distribuição espacial dirigido a tais espécies. Meirelles \& Luiz (1995) ressaltaram a necessidade de trabalhos com esta abordagem, uma vez que investigações nesta linha são raras nas áreas de Cerrado.

Anadenanthera falcata apresentou os maiores valores de Dominância Relativa (DoR) e Índice do Valor de Importância (IVI), sendo que a DoR (31,75\%) foi aproximadamente 4,5 vezes maior do que aquela apresentada por Myrcia guianensis (7,29\%). Tal diferença está, obviamente, mais relacionada ao porte da primeira espécie (maior área basal) do que ao número de indivíduos, pois Myrcia guianensis foi a mais abundante e com os maiores valores de DR.

Ouratea spectabilis e Copaifera langsdorffii também são destaque em outros estudos de cerrados em São Paulo entre as espécies com os maiores valores de IVI (Pagano et al. 1989; Toledo Filho et al. 1989; Durigan et al. 1994; Durigan et al. 1997). C. langsdorffii também figura em trabalhos fitossociológicos de matas ripárias como uma das espécies mais importantes (Carvalho et al. 1995).

A Fig. 1 mostra que a distribuição de classes de diâmetro dos indivíduos da área estudada forma um "J" invertido, estando a maioria dos indivíduos $(67,88 \%)$ concentrada na primeira classe. De acordo com Silva Júnior \& Silva

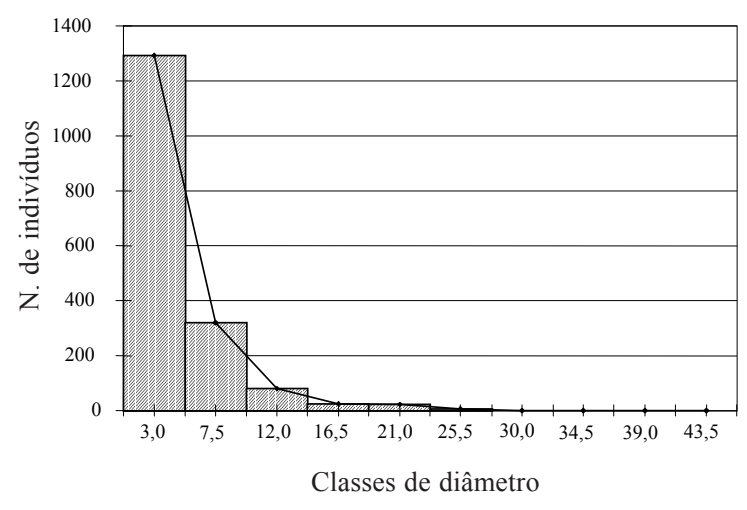

Figura 1. Classes de diâmetro $(\mathrm{cm})$ dos indivíduos da área de cerrado stricto sensu na Gleba Cerrado Pé-de-Gigante. Classes fixas de 4,5cm. 1) 1 a 5,5;2) 5,6 a 10 ; 3) 10,1 a $14,5$; 4) 14,6 a $19 ; 5) 19,1$ a 23,5 ; 6) 23,6 a 28 ; 7 ) 28,1 a $32,5$; 8$) 32,6$ a 37 ; 9) 37,1 a 41,5 ; 10) 41,6 a 46 .

(1988), maior número de indivíduos nas classes inferiores pode indicar que a maioria das populações estaria na fase inicial do estabelecimento. Mas os autores ainda ressaltam que, quando se trata de cerrado, deve-se lembrar que algumas espécies apresentam menor porte, por esta ser sua potencialidade genética. Nota-se ainda que a razão "q" não é constante, evidenciando condição não balanceada. Harper (1977) afirmou que a maioria das florestas não apresenta distribuição balanceada, mas convergem para tal.

Ao analisar a Fig. 2, pode-se observar que a maioria dos indivíduos (72,3\%) está entre 1 e $3 \mathrm{~m}$ de altura. Número pouco expressivo de indivíduos nos segmentos de maiores alturas pode indicar que essa vegetação não forma um dossel regular, e sim que os indivíduos mais altos têm alturas diferentes. Isto é o que ocorre na área estudada, onde o dossel não forma um telhado horizontal contínuo, como era esperado para o cerrado stricto sensu.

Nota-se na Fig. 3 que apenas duas espécies alcançaram $8 \mathrm{~m}$ de altura Anadenanthera falcata e Copaifera langsdorffii, embora algumas outras espécies se destaquem como emergentes: Qualea dichotoma, Diptychandra aurantiaca e Bredemeyera floribunda. 


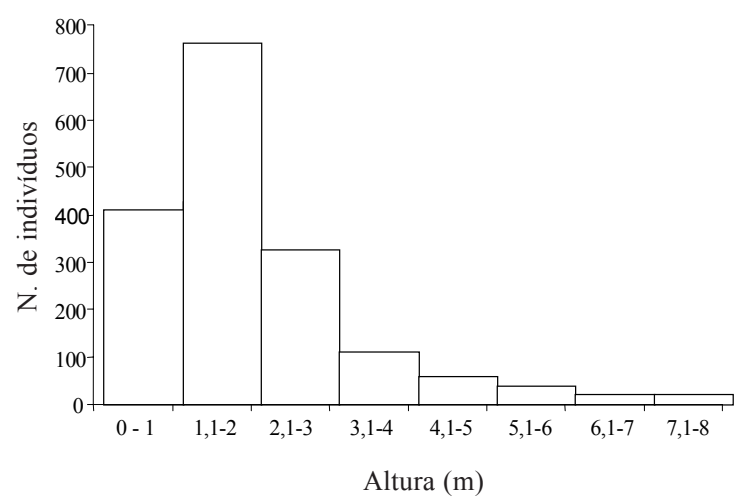

Figura 2. Altura $(\mathrm{m})$ dos indivíduos amostrados na área de cerrado stricto sensu na Gleba Cerrado Pé-de-Gigante.

A presente investigação ressalta as seguintes características da área em questão: trata-se de um fragmento de cerrado s.s. cuja maioria dos indivíduos concentra-se no estrato inferior a $3 \mathrm{~m}$ de altura, com algumas espécies emergentes, que chegam a $8 \mathrm{~m}$ ou mais, de destacada importância na estrutura da comu- nidade, pois figuram entre as de maior IVI, como exemplificado por Anadenanthera falcata pela combinação dos expressivos números de indivíduos e área basal. Tal figura, entretanto, não mostra dois estratos bem distintos, um arbóreo e outro arbustivo, como a definição de Eiten (1994) para o cerrado s.s. A área em questão apresenta grande número de espécimes em estádio jovem, muitos deles espécies componentes do estrato arbustivo. Há grande número de espécies comuns a outras áreas de Cerrado no Estado de São Paulo: Stryphnodendron adstringens, Pterodon pubescens, Hymenaea stignocarpa, Copaifera langsdorffii, Xylopia aromatica, Bowdichia virgilioides, Caryocar brasiliense e Qualea grandiflora, muitas delas de uso popular, inclusive medicinal, como informam Vieira \& Martins (2000).

Os resultados acrescentam dados que poderão subsidiar as ações de manutenção e preservação da vegetação em remanescentes do Cerrado no Estado de São Paulo.

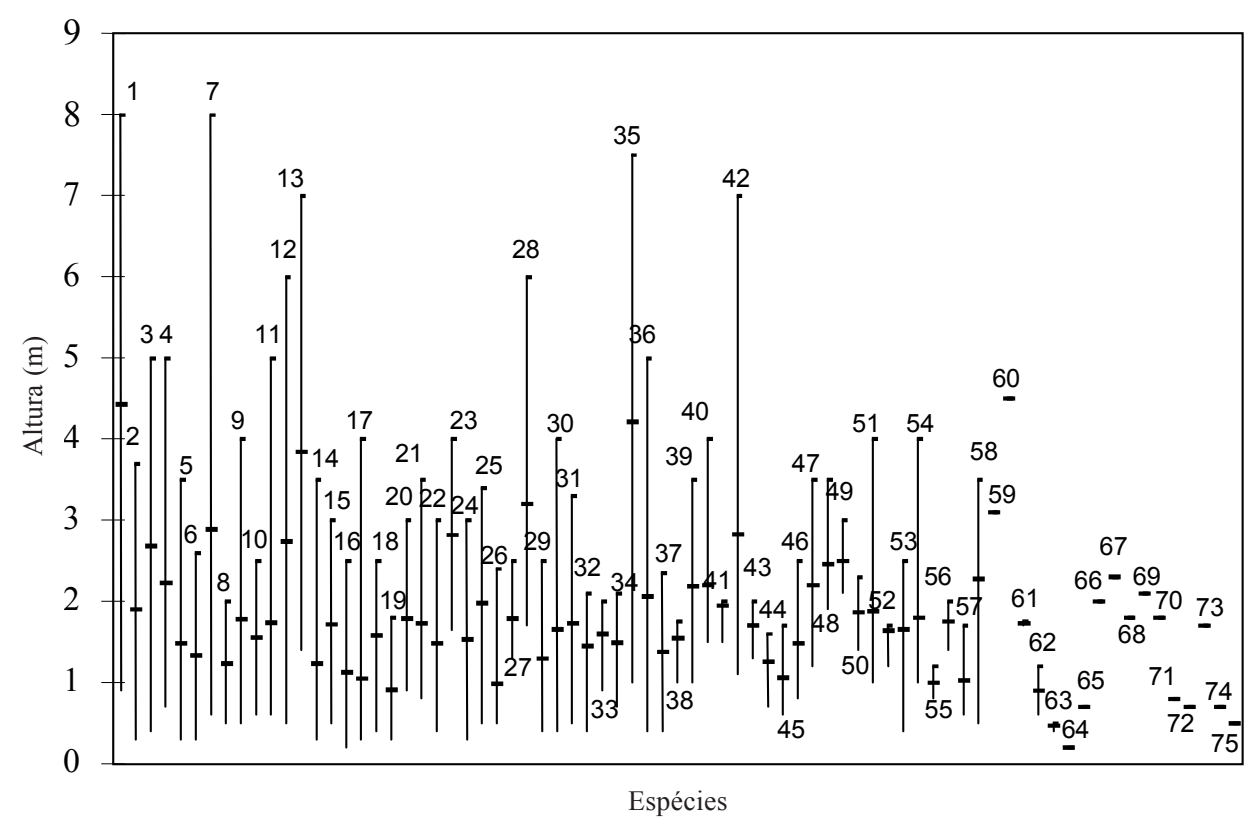

Figura 3. Espaço vertical ocupado pelas espécies amostradas, ordenadas de forma decrescente de Índice do Valor de Importância (IVI). A numeração das espécies segue a Tabela 1. 


\section{Agradecimentos}

Ao CNPq, pela concessão de bolsa de Iniciação Científica à primeira Autora; ao Prof. Dr. Fernando Roberto Martins, pelas valiosas sugestões no decorrer da investigação; a Daniel de Wet, pela revisão do abstract.

\section{Referências bibliográficas}

Bitencourt, M. D.; Mesquita Jr., H. N.; Mantovani, W.; Batalha, M. A. \& Pivello, V. R. 1997. Identificação de fisionomias de cerrado com imagem índice de vegetação. Pp. 316-320. In: L. L. Leite. \& C. H. Saito (orgs.). Contribuição ao Conhecimento Ecológico do Cerrado. Editora Universidade de Brasília, Brasília.

Carvalho, D. A.; Oliveira-Filho, A. T.; Vilela, E. A. \& Gavilanes, M. L. 1995. Estrutura fitossociológica de mata ripária do alto do Rio Grande (Bom Sucesso, Estado de Minas Gerais). Revista Brasileira de Botânica 18(1): 39-49.

Castro, A. A. J. F.; Martins, F. R. \& Fernandes, A. G. 1998. The woody flora of cerrado vegetation in the State of Piauí, Northeastern Brazil. Edinburg Journal of Botany 55(3): 455-472.

Castro, A. A. J. F.; Martins, F. R.; Tamashiro, J. Y. \& Shepherd, G. J. 1999. How rich is the flora of Brazilian Cerrados? Annals of the Missouri Botanical Garden 86(2): 192-224.

Coutinho, L. M. 1978. O conceito de Cerrado. Revista Brasileira de Botânica 1(1): 17-23.

Coutinho, L. M. 1982. Ecological effects of fire in Brazilian Cerrado. Pp. 273-291. In: B. J. Huntley \& B.H. Walker (eds.). Ecology of Tropical Savannas. Springer-Verlag, Berlin.

Curtis, J. T. \& McIntosh R. P. 1950. The interrelations of certain analytic and synthetic phytosociological characters. Ecology 31: 434-455.

Dias, M. C.; Vieira, A. O. S.; Nakajima, J. N.; Pimenta, J. A. \& Lobo, P. C. 1998. Composição florística e fitossociológica do componente arbóreo das florestas ciliares do rio Iapó, na bacia do rio Tibagi, Tigabi, PR. Revista Brasileira de Botânica 21(2): 183-195.

Durigan, G.; Franco, G. A. D. C.; Pastore, J. A. \& Aguiar, O. T. 1997. Regeneração natural da vegetação de cerrado sob floresta de Eucalyptus citriodora. Revista do Instituto Florestal 9(1): 71-85.

Durigan, G.; Leitão Filho, H. F. \& Rodrigues, R. R. 1994. Phytosociology and structure of a frequently burnt cerrado vegetation in SE- Brazil. Flora 189: 153-160.
Durigan, G.; Saraiva, I. R.; Amadeu Garrido, L. M. A.; Garrido, M. A. O. \& Peche Filho, A. 1987. Fitossociologia e evolução da densidade da vegetação do cerrado de Assis, SP. Boletim Técnico do Instituto Florestal 41(1): 59-78.

Eiten, G. 1994. Vegetação do Cerrado. Pp. 17-73. In: M. N. Pinto (org.). Cerrado: Caracterização, Ocupação e Perspectivas. Editora Universidade de Brasília, Brasília.

Felfili, J. M. \& Silva Júnior, M. C. 1993. A comparative study of cerrado (stricto sensu) vegetation in Central Brazil. Journal of Tropical Ecology 9: 277-289.

Felfili, J. M.; Silva Júnior, M. C. \& Nogueira, P. E. 1998. Levantamento da vegetação arbórea na região de Nova Xavantina, MT. Boletim do Herbário Ezechias Paulo Heringer 3: 63-81.

Felfili, J. M.; Silva Júnior, M. C.; Rezende, A. V.; Machado, J. W. B.; Walter, B. M. T.; Silva, P. E. N. \& Hay, J. D. 1993. Análise comparativa da florística e fitossociologia da vegetação arbórea do cerrado sensu stricto na Chapada Pratinha, DF-Brasil. Acta Botanica Brasilica 6(1): 27-46.

Guarim-Neto, G.; Guarim, V. L. M. S. \& Prance, G. T. 1994. Structure and floristic composition of the trees of an area of cerrado near Cuiabá, Mato Grosso, Brazil. Kew Bulletin 49(3): 499-509.

Harper, T. L. 1977. Population biology of plants. Academic Press, New York.

Heringer, E. P.; Barroso, G. M.; Rizzo, J. A. \& Rizzini, C. T. 1977. A flora do Cerrado. Pp. 211-232. In: M. G. Ferri (coord.). IV Simpósio sobre o cerrado. EDUSP e Ed. Itatiaia, São Paulo e Belo Horizonte.

Köppen, W. 1948. Climatologia. Ed. Fondo de Cultura Económica, México.

Leitão Filho, H. F. 1992. A flora arbórea dos Cerrados do Estado de São Paulo. Hoehnea 19(1/2): 151-163.

Magurran, A. E. 1988. Ecological diversity and its measurement. Chapman and Hall, London.

Martins, F. R. 1993. Estrutura de uma floresta mesófila. 2a Edição. Editora da Unicamp, Campinas.

Meirelles, M. L. \& Luiz, A. J. B. 1995. Padrões espaciais de árvores de um cerrado em Brasília, DF. Revista Brasileira de Botânica 18(2): 185-189.

Mendonça, R. C.; Felifili, J. M.; Walter, B. M. T.; Silva Júnior, M. C.; Rezende, A. B.; Filgueiras, T. S. \& Nogueira, P. E. 1998. Flora Vascular do Cerrado. Pp. 289-556. In: S. M. Sano \& S. P. Almeida (eds.). Cerrado: ambiente e flora. EMBRAPA - CPAC, Planaltina. 
Oliveira-Filho, A. T. \& Ratter, J. A. 1995. A study of the origin of central Brazilian forests by the analysis of plant species distribution patterns. Edinburg Journal of Botany 52(2): 141-194.

Oliveira-Filho, A. T.; Shepherd, G. D.; Martins, F. R. \& Stubblebine, W. H. 1989. Environmental factors affecting physiognomic and floristic variation in an area of cerrado in Central Brazil. Journal of Tropical Ecology 5: 413-431.

Pagano, S. N.; Cesar, O. \& Leitão Filho, H. F. 1989. Estrutura fitossociológica do estrato arbustivoarbóreo da vegetação de cerrado da área de proteção ambiental (APA) de Corumbataí, estado de São Paulo. Revista Brasileira de Biologia 49(1): 49-59.

Piccolo, A. L. G.; Thomazini, L. I.; Massa, C. S.; Cesar, O.; Pagano, S. N.; Moraes, J. A. P. V. \& Amaral, H. 1971. Aspecto fitossociológico de uma reserva de cerrado. Revista de Agricultura 46: 81-92.

Pivello, V. R. \& Coutinho, L. M. 1996. A qualitative successional model to assist in the management of Brazilian cerrados. Forest Ecology and Management 87(1-3): 127-138.

Pivello, V. R.; Bitencourt, M. D.; Mantovani, W.; Mesquita Jr., H. N.; Batalha, M. A. \& Shida, C. 1998. Proposta de zoneamento ecológico para a reserva de cerrado Pé-de-Gigante (Santa Rita do Passa Quatro, SP). Revista Brasileira de Ecologia 2: 108-118.

Pivello, V. R.; Bitencourt, M. D.; Mantovani, W.; Mesquita Jr., H. N. \& Batalha, M. A. 1999. Banco de dados em SIG para Ecologia aplicada: exemplo do Cerrado Pé-de-Gigante, SP. Caderno de Informações Georreferenciadas 1:http:// www.cpa.unicamp.br/revista/cigv1n3a4.html.

Ratter, J. A.; Bridgewater, S.; Atkinson, R. \& Ribeiro, J. F. 1996. Analysis of the floristic composition of the Brazilian cerrado vegetation II: comparison of the woody vegetation of 98 areas. Edinburg Journal of Botany 53: 153-180.

Ratter, J. A. \& Dargie, T. C. D. 1992. An analysis of the floristic composition of 26 cerrado areas in Brazil. Edinburg Journal of Botany 49(2): 235-250.

Ratter, J.A.; Leitão Filho, H.F.; Argent, G.; Gibbs, P.E.; Semir, J.; Shepherd, G. \& Tamashiro, J. 1988. Floristic composition and community structure of a Southern cerrado area in Brazil. Notes of the Royal Botanical Garden of Edinburg 45(1): 137-151.

Ratter, J. A.; Ribeiro, J. F. \& Bridgewater, S. 1997. The Brazilian cerrado vegetation and threats to its biodiversity. Annals of Botany 80(3): 223-230.
Ribeiro, J. F.; Silva, J. C. S. \& Batmanian, G. J. 1985. Fitossociologia de tipos fisionômicos de cerrado em Planaltina, DF. Revista Brasileira de Botânica 8(2): 131-142.

Ribeiro, J. F. \& Walter, B. M. T. 1998. Fitofisionomias do bioma cerrado. Pp. 89-166. In: S. M. Sano \& S. P. Almeida (eds.), Cerrado: ambiente e flora. EMBRAPA - CPAC, Planaltina.

Rizzini, C. T. 1997. Tratado de fitogeografia do Brasil. $2^{\mathrm{a}}$ Edição. Âmbito Cultural Edições Ltda, Rio de Janeiro.

Silberbauer-Gottsberger, I. \& Eiten, G. 1983. Fitossociologia de um hectare de cerrado. Brasil Florestal 54: 55-70.

Silva Júnior, M. C.; Barros, N. F. \& Cândido, F. 1987. Relações entre parâmetros do solo e da vegetação de cerrado na Estação Florestal de Experimentação de Paraopeba, MG. Revista Brasileira de Botânica 10(2): 125-137.

Silva Júnior, M. C. \& Silva, A. F. 1988. Distribuição dos diâmetros dos troncos das espécies mais importantes do cerrado na Estação Florestal de Experimentação de Paraopeba (EFLEX), MG. Acta Botanica Brasilica 2(1-2): 107-126.

Tabarelli, M. \& Mantovani, W. 1999. A riqueza de espécies arbóreas na floresta atlântica de encosta no Estado de São Paulo (Brasil). Revista Brasileira de Botânica 22(2): 217-223.

Terborgh, J. \& Andresen, E. 1998. The composition of Amazonian forests: patterns at local and regional scales. Journal of Tropical Ecology 14(5): 645-664.

Toledo Filho, D. V.; Leitão Filho, H. F. \& Rodrigues, T. S. 1984. Composição florística de área de cerrado em Moji Mirim (SP). Boletim Téenico do Instituto Florestal 38(2): 165-175.

Toledo Filho, D. V.; Leitão Filho, H. F. \& Shepherd, G. 1989. Estrutura fitossociológica da vegetação de cerrado em Mogi-Mirim (SP). Revista do Instituto Florestal 1(2): 1-11.

Uhlmann, A.; Galvão, F. \& Silva, S. M. 1998. Análise da estrutura de duas unidades fitofisionômicas de savana (cerrado) no Sul do Brasil. Acta Botanica Brasilica 12(3): 231-247.

van den Berg, E. \& Oliveira-Filho, A. T. 2000. Composição florística e estrutura fitossociolótica de uma floresta ripária em Itutinga, $\mathrm{MG}$, e comparação com outras áreas. Revista Brasileira de Botânica 23(3): 231-253.

Vieira, R. F. \& Martins, M. V. M. 2000. Recursos genéticos de plantas medicinais do cerrado: uma compilação de dados. Revista Brasileira de Plantas Medicinais 3(1): 13-36. 